\title{
A Mediterranean Diet-Based Lifestyle Intervention in Inflammatory Bowel Disease Patients with Quiescent Disease; A Pilot Study
}

\author{
Molendijk $\mathrm{I}^{1 \#}$, Van Lingen $\mathrm{E}^{1 * \#}$, Steenhuis $\mathbf{M}^{1}$, Van der Marel $\mathrm{S}^{2}$, Van der Meulen de Jong $\mathrm{AE}^{1}$, \\ Wilmsen $\mathbf{N}^{3}$, Ernst-Stegeman $\mathrm{A}^{3}$ and Maljaars PWJ ${ }^{1}$ \\ ${ }^{1}$ Department of Gastroenterology and Hepatology, Leiden University Medical Center, Netherlands \\ ${ }^{2}$ Department of Gastroenterology and Hepatology, Haaglanden Medical Center (HMC), Netherlands \\ ${ }^{3}$ Voeding Leeft, Netherlands \\ ${ }^{*}$ Author Molendijk I and van Lingen E contributed equally
}

*Corresponding author: E Van Lingen, Department of Gastroenterology and Hepatology, Leiden University Medical Center, Netherlands.

To Cite This Article: Molendijk I, Van Lingen E, Steenhuis M, Van der Marel S, Van der Meulen de Jong AE, et al., A Mediterranean Diet-Based Lifestyle Intervention in Inflammatory Bowel Disease Patients with Quiescent Disease; A Pilot Study. Am J Biomed Sci \& Res. 2021 - 13(1). AJBSR. MS.ID.001830. DOI: 10.34297/AJBSR.2021.13.001830.

Received: 眥 March 26, 2021; Published: 拝 June 01, 2021

\begin{abstract}
Aim: Evidence is emerging that a Mediterranean diet (MeD) can modulate the immune system and restore the gut epithelial barrier, thereby ameliorating inflammatory bowel disease (IBD)-related symptoms and quality of life (QoL) in IBD patients. We investigated the effects of a MeDbased lifestyle intervention on tolerance, IBD-related symptoms, QoL and inflammation in IBD patients with quiescent disease.

Methods: 39 patients with quiescent IBD were included to follow the MeD-based lifestyle intervention of Voeding Leeft, consisting of three full-day live group sessions and professional guidance during three months. Patients filled in validated questionnaires to evaluate their current diet (Mediterranean Diet Serving Score (MDSS)) and to determine their food-related QoL (FRQoL), disease-related QoL (short IBD questionnaire (sIBDQ)), the severity of fatigue and IBD-related disability (IBD disability index (IBD-DI)). Body Mass Index (BMI) was calculated and the hand grip strength (HGS) was measured, like C-reactive protein (CRP) in the blood and Calprotectin (FCP) in the feces.
\end{abstract}

Results: In quiescent IBD patients, $69 \%$ was able to adhere to the MeD-based lifestyle intervention. The improvement in MDSS was correlated to the increase in QoL, but this effect was only observed in patients with Crohn's disease (CD), not with ulcerative colitis (UC). CRP and BMI decreased during the study period.

Conclusion: A MeD-based lifestyle intervention was well-tolerated in patients with quiescent IBD. At 6 months patients reported less fatigue, patients had an improved quality of life and a lower BMI with an improved handgrip strength. This study underlines the importance of nutrition in the management of IBD.

Keywords: Mediterranean Diet; Lifestyle; Inflammatory Bowel Disease; Quality of life

Abbreviations: CD: Crohn's Disease, UC: Ulcerative Colitis, CRP: C-Reactive Protein HGS: Hand Grip Strength; IBD: Inflammatory Bowel Disease

\section{Introduction}

Nutrition and food are important topics for inflammatory bowel disease (IBD) patients [1]. More than half of the IBD patients indicated in two different studies that diet is essential for them to maintain quiescent disease [2,3]. In quiescent IBD, $77 \%$ of the patients avoid certain foods such as spicy foods, foods with a high fat content and carbonated beverages, because they believe intake of those foods will trigger symptoms such as abdominal pain, bloating and diarrhea [3] Dietary patterns should not only be important to IBD patients, but also to their treating physicians. In 
recent decades, consumption of processed and industrialized foods has increased. The so-called Western diet, which consists of a low intake in fiber and n-3 polyunsaturated fatty acids (PUFA) and a high intake of refined sugars and n-6 PUFA, increases the risk to develop IBD. For instance, Crohn's disease (CD) is 50\% less likely to occur when consuming a healthy diet including fish, fruits and vegetables decades before the onset of the disease [4].

Not only the onset but also the disease course of IBD may be influenced by what patients consume. For example, a low n-6/n-3 PUFA ratio is associated with a higher level of disease activity [57]. There is growing evidence that a Western diet leads to a less diverse gut microbiome which can lead to a decrease in intestinal barrier function. IBD patients already have a disturbed intestinal barrier function even when only little inflammation is present, which is associated with gastrointestinal symptoms and risk of a disease flare $[8,9]$. Lower diversity of the microbiome composition results in a decreased production of short-chain fatty acids (SCFA). These SCFA are important for maintaining homeostasis in the colon, and thereby an anti-inflammatory state [10]. In addition, the increased salt intake observed in a Western dietary pattern induces pathogenic T helper 17 (Th17) cells which play an important role in the pathogenesis of $\mathrm{CD}[11,12]$. These data suggest that both wellbeing but also disease control could be improved by means of dietary manipulation [13]. The Mediterranean diet (MeD) is able to promote a healthy gut microbiome, restore the intestinal barrier function and decrease systemic inflammation [1]. The MeD is rich in fruits, vegetables, fibers and n-3 PUFA, and low in refined sugars and saturated, monounsaturated and n-6 PUFA. In cardiovascular and metabolic diseases, excellent results have been made by introducing the MeD $[14,15]$. In the landmark Predimed paper, Estruch et al. demonstrated that introduction of a MeD reduced the incidence of major cardiovascular events and also trials in rheumatic diseases have shown that a MeD reduced symptoms and biomarkers of inflammation (i.e. C-reactive protein (CRP)) [16]. More recently and specifically in the IBD-field, Chicco et al. [17] found that the adherence to a MeD significantly reduce disease activity and inflammation-related biomarkers in IBD patients [17] Furthermore, Papada et al. [19] found that adherence to a MeD is associated with improved quality of life in CD patients [18]. On the other hand, Vrdoljak et al. reported a very low MeD adherence in IBD patients; patients are indeed willing to extend their nutritional knowledge, but proper educational programs are needed [19] With this in mind, we aimed to investigate how a Mediterranean dietbased lifestyle intervention would be tolerated by IBD-patients in remission when following a specific lifestyle program. Secondly, we investigated the effects of the intervention on quality of life (QoL) and inflammatory markers (CRP and fecal calprotectin (FCP)).

\section{Methods}

Study Population: In March 2018, 39 IBD patients with quiescent disease were included to follow the MeD-based lifestyle intervention of Voeding Leeft at the department of Gastroenterology and Hepatology at the Leiden University Medical Center (LUMC).

Patient Characteristics: Patients were eligible for inclusion when they had quiescent IBD, defined as a FCP $<150 \mu \mathrm{g} / \mathrm{g}$ and/ or a simple endoscopic score-CD (SES-CD) $<5$ or an endoscopic MAYO score $<1$ in case of UC. Exclusion criteria were age $<18$ years, symptoms due to stenotic disease, a history of an eating disorder (DSM IV), a current healthy lifestyle that resembled the MeD, not being able to adhere to MeD and not being able to join the three live days of the intervention program. At baseline and at the end of follow-up, patients visited the LUMC. Demographic information (age, gender, type and duration of IBD, current medication and history of surgery for IBD, concomitant disease and intoxications) was collected from all patients. Blood was drawn for CRP measurements and FCP levels were measured. The Mediterranean Diet Serving Score (MDSS; range 0-24) was used to evaluate current diet at the end of follow-up: a score $>14$ at baseline was classified as already a $\mathrm{MeD}$ and was therefore an exclusion criterium. Disease activity scores were determined with the Harvey-Bradshaw index (HBI) in case of CD and the partial Mayo score (PMS) in case of UC. Patients filled in questionnaires to determine their food-related quality of life (FRQoL), disease-related quality of life (short IBD questionnaire (SIBDQ)), the severity of fatigue and IBD-related disability (IBD disability index (IBD-DI)). Body mass index (BMI) was calculated and hand grip strength (HGS) was measured in kilograms with a Jamar Hand Dynamometer.

Lifestyle Program and Diet: The Mediterranean dietbased lifestyle intervention for IBD patients is one of the lifestyle programs for people with a chronic disease developed by Voeding Leeft. Another highly successful program from Voeding Leeft is Reverse Diabetes 2 Now. The IBD lifestyle program is an intensive group program that consists of three live days. During these days participants meet the other included IBD patients and are educated on the elements of a healthy diet and how to implement these in daily life. The recommended diet consists of ample amount of unprocessed foods, mainly plant-based and only small-moderate amount of animal foods. When compared to other well-known diets, the prescribed diet corresponds very closely to the Mediterranean diet. Therefore, in this manuscript the diet is referred to as MeD. Our diet resembles the Mediterranean diet in that it was as unprocessed as possible and rich in olive oil, vegetables, legumes, nuts and seeds. Only whole grains, fish and smaller amounts of (red) meat and dairy were consumed. It differs from the Mediterranean diet 
because patients were only allowed to eat three meals a day without snacking in between. Furthermore, no alcohol and processed meats were allowed. The consumption of pre- and probiotic foods was encouraged as well as foods rich in calcium, zinc, iron, potassium, B-vitamins, magnesium and selenium. The dietary guidelines of the IBD program are based on the recommendations of the Dutch Health Council (Richtlijnen Goede Voeding), the nutritional guidelines of the WHO and FDA and on additional research on nutrition in IBD.

Next to the three live group sessions, participants received a workbook with recipes, a shopping list and a one month meal plan, and professional and intensive (online) guidance through an online peer and support community. This to encourage the participants to adhere to the diet. Inspirational content is regularly shared and discussed online, questions are answered, and they had access to all background information at any time. Participants were gradually introduced to the MeD by replacing one meal with a meal from the program each week, until they consume 3 meals a day in line with the prescribed MeD. After three months the active part of the Mediterranean diet-based lifestyle intervention ends, however participants are encouraged to continue their new lifestyle after the program. For example, they can still access the online community and they can keep in touch with their peers. Patients were followedup for three months after the end of the program.

Table 1: Baseline characteristics.

\section{Statistical Analysis}

Statistical analyses were performed using IBM SPSS Statistics, version [23]. Descriptive statistics were used for baseline characteristics (mean (SD), median (IQR) or number (\%)). Measurements at baseline and at the end of follow-up were compared with a paired samples T-test and a Chi-square test, a Wilcoxon signed rank test was used when data was not normally distributed. A p-value $<0.05$ was considered statistically significant.

Informed consent was obtained from all participants prior to inclusion in the study. This research project was reviewed and approved by the Medical Ethical Committee in the LUMC, with reference number NL63643.058.

\section{Results}

Patients: In this open label pilot study, 39 patients with a mean age of 46.4 years (SD 12.2) were included. (Table 1) 25 patients $(64.1 \%)$ were female and 26 patients $(66.7 \%)$ had CD. Based on FCP or CRP, 38/39 patients were in remission. The one patient that had a FCP $>250 \mu \mathrm{g} / \mathrm{g}$ had a colonoscopy demonstrating endoscopic remission, and was therefore allowed to participate. 14 patients (35.9\%) were overweight (BMI $25-29.99 \mathrm{~kg} / \mathrm{m} 2)$, and 10 patients $(25.6 \%)$ were obese $\left(\mathrm{BMI}>30 \mathrm{~kg} / \mathrm{m}^{2}\right)$. Two patients also had a diagnosis of type 2 diabetes, 6 patients had a diagnosis of hypertension and 1 patient had dyslipidemia.

\begin{tabular}{|c|c|}
\hline & IBD patients $(n=39)$ \\
\hline Age, mean (SD), y & $46.4(12.2)$ \\
\hline Men, N (\%) & $14(35.9)$ \\
\hline Crohn's disease, N (\%) & $26(66.7)$ \\
\hline \multicolumn{2}{|l|}{ Age at Diagnosis, $\mathrm{N}(\%)$} \\
\hline $\mathrm{A} 1(<16$ years $)$ & $1(3.8)$ \\
\hline A2 (17 - 40 years) & $23(88.5)$ \\
\hline A3 (> 40 years) & $2(7.7)$ \\
\hline \multicolumn{2}{|l|}{ Location of disease, $\mathrm{N}(\%)$} \\
\hline L1 (ileal disease) & $13(50.0)$ \\
\hline L2 (colonic disease) & $5(19.2)$ \\
\hline L3 (ileal and colonic disease) & $8(30.8)$ \\
\hline L4 (disease proximal of ileum) & $0(0)$ \\
\hline Perianal disease $^{a}$ & $2(7.7)$ \\
\hline \multicolumn{2}{|l|}{ Disease Behaviora ${ }^{\mathrm{a}}$ (\%) } \\
\hline B1 (non-stricturing, non-penetrating) & $14(53.8)$ \\
\hline B2 (stricturing) & $9(34.6)$ \\
\hline B3 (penetrating) & $2(7.7)$ \\
\hline Ulcerative colitis, $\mathrm{N}(\%)$ & $13(33.3)$ \\
\hline \multicolumn{2}{|l|}{ Extension of disease, $\mathrm{N}(\%)$} \\
\hline E1 (rectal disease) & $4(30.8)$ \\
\hline E2 (distal from splenic flexure) & $6(46.2)$ \\
\hline E3 (proximal from splenic flexure) & $3(23.1)$ \\
\hline
\end{tabular}




\begin{tabular}{|c|c|}
\hline Severity of Disease ${ }^{\mathrm{b}}, \mathrm{N}(\%)$ & \\
\hline S0 (remission) & $9(69.2)$ \\
\hline S1 (mild) & $4(30.8)$ \\
\hline S2 (moderate) & $0(0)$ \\
\hline S3 (severe) & $0(0)$ \\
\hline Duration of IBD, mean (SD), y & $16.7(12.5)$ \\
\hline Medication for IBD, N (\%) & $26(66.7)$ \\
\hline 5-aminosalicylic acid (5-ASA) & $8(20.5)$ \\
\hline Thiopurine & $8(20.5)$ \\
\hline Methotrexate & $1(2.6)$ \\
\hline Biological & $14(35.9)$ \\
\hline Budesonide & $0(0)$ \\
\hline Prednisone & $1(2.6)$ \\
\hline Bowel resection for IBD, N (\%) & $15(38.5)$ \\
\hline \multicolumn{2}{|l|}{ Concomitant disease, $\mathrm{N}(\%)$} \\
\hline Diabetes & $2(5.1)$ \\
\hline Hypertension & $6(15.4)$ \\
\hline High lipid spectrum & $1(2.6)$ \\
\hline Smoker, N (\%) ${ }^{a}$ & $0(0)$ \\
\hline Alcohol consumption, $\mathrm{N}(\%)^{\mathrm{a}}$ & $13(33.3)$ \\
\hline
\end{tabular}

Note: ${ }^{a} 1$ missing value; bbased on clinical score; IBD Inflammatory bowel disease; N number; SD standard deviation; y years

\section{Effect of a MeD-based lifestyle intervention}

Out of 39 patients, 27 (69\%) completed the MeD-based lifestyle intervention. Eleven patients reported why they dropped out, some of them reported more than one reason. Eight patients found the intervention program too much effort or did not have the time or motivation to implement it into their daily life, 3 patients missed some group sessions and/or individual sessions and therefore dropped-out, 1 patient was too tired to complete the program and 1 patient reported abdominal complaints (without biochemical signs of a flare) as a reason to drop out. In Table 2, the completers and dropouts were compared at baseline. Median IBD complaints, as measured by the HBI or PMS, was significantly lower in completers versus dropouts. Both the SIBDQ and IBD DI questionnaires differed between these groups, whereas FRQoL or MDSS at baseline did not differ between groups. In total, 25 patients improved their dietary intake, defined as an increase in the MDSS by at least 1 point, the mean improvement in MDSS was 3.0 (SD 3.75). The results of the MDSS showed that ingestion of olive oil $(p=0.006)$, nuts ( $p=$ $0.004)$, fish ( $p=0.014)$ and poultry $(p<0.001)$ increased, whereas ingestion of potatoes $(\mathrm{p}<0.001)$, dairy $(\mathrm{p}<0.001)$ and alcohol $(0=$ 0.007) decreased (Chi square, data not shown).

Table 2: Comparing completers and dropouts at baseline.

\begin{tabular}{|c|c|c|c|}
\hline & Completers (n= 27) & Dropouts (n = 12) & p-value \\
\hline Age, mean (SD), y & $47.2(12.4)$ & $44.5(12.1)$ & 0.53 \\
\hline Male, N (\%) & $9(33.3)$ & $5(41.7)$ & 0.62 \\
\hline IBD type (CD, N (\%)) & $18(66.7)$ & $8(66.7)$ & 0.0 \\
\hline HBI, median (IQR) & $2(1-4.25)$ & $4.5(3-7)$ & 0.026 \\
\hline pMS, median (IQR) & $1(0-2)$ & $1.5(0-3)$ & 0.71 \\
\hline Body weight, mean (SD), kg & $82.6(20.8)$ & $86.9(22.1)$ & 0.56 \\
\hline BMI, mean (SD) & $27.3(6.2)$ & $28.1(4.7)$ & 0.68 \\
\hline Fatigue, mean (SD) & $5.2(2.1)$ & $4.7(2.5)$ & 0.49 \\
\hline Baseline MDSS, mean (SD) & $9.7(2.8)$ & $9.7(3.8)$ & 0.96 \\
\hline sIBDQ mean (SD) & $52.9(7.8)$ & $43.1((11.2)$ & 0.005 \\
\hline FRQoL, mean (SD) & $86(22.9)$ & $74.8(19.8)$ & 0.17 \\
\hline IBD-DI, mean (SD) & $24.9(12.3)$ & $39.5(12.4)$ & 0.004 \\
\hline
\end{tabular}

Note: BMI: Body Mass Index; HBI: Harvey-Bradshaw index; CD: Crohn's Disease; FRQoL: Food Related Quality of Life; IBD: Inflammatory Bowel Disease; IBD-DI: IBD Disability Index; IQR: Interquartile Range; kg: kilograms; MDSS: Mediterranean Diet Serving Score; N: Number; Pms: Partial Mayo Score; SD: Standard Deviation; sIBDQ: Short IBD Questionnaire; y: Years. 
As shown in Table 3, the mean dietary quality improved between baseline and six months (9.7 (SD 2.9) vs 12.8 (SD 2.9), p $<0.001$ ). CRP decreased from baseline to 6 months (3.6 (SD 2.6) vs 2.6 (SD 2.6), $\mathrm{p}=0.037$ ), whereas FCP did not change (67 (SD 68) vs 73 (SD 99), $\mathrm{p}=0.78$ ). There was a trend towards an improvement in remission status (Wilcoxon signed rank test, $\mathrm{p}=0.053$ ) (Table 3 ), but this was mainly due to the effect in patients with $\mathrm{CD}(\mathrm{p}=$
0.050). When assessing only the completers, remission status improved both in the overall analysis $(\mathrm{p}<0.001)$ as in the analysis in CD patients $(\mathrm{p}<0.001)$ (data not shown). During the study, 37/39 patients did not show any signs of a flare. One patient moved to another part of the Netherlands and changes in remission status or medication could not be followed up.

Table 3: Effects of the Mediterranean diet-based lifestyle intervention.

\begin{tabular}{|c|c|c|c|c|c|c|c|c|c|}
\hline & \multicolumn{3}{|c|}{ Overall $(n=39)$} & \multicolumn{3}{|c|}{$C D(n=26)$} & \multicolumn{3}{|c|}{ UC $(n=13)$} \\
\hline & $t=0$ & $\mathrm{t}=6 \mathrm{mts}$ & $\mathrm{p}$ & $t=0$ & $\mathrm{t}=6 \mathrm{mts}$ & $\mathrm{p}$ & $t=0$ & $\mathrm{t}=6 \mathrm{mts}$ & $\mathrm{p}$ \\
\hline MDSS mean (SD) & $9.7(2.9)$ & $12.8(2.9)$ & $<0.001^{\mathrm{a}}$ & $9.2(2.7)$ & $13.3(2.2)$ & $<0.001$ & $10.6(2.9)$ & $11.9(3.6)$ & 0.34 \\
\hline CRP mean (SD) & $3.6(3.5)$ & $2.6(2.6)$ & $0.037^{a}$ & $3.6(3.7)$ & $2.3(2.5)$ & 0.024 & $3.4(3.1)$ & $3.2(2.9)$ & 0.79 \\
\hline FCP mean (SD) & $67(68)$ & 73 (99) & $0.78^{\mathrm{a}}$ & $74.5(76.9)$ & $69.3(85.4)$ & 0.81 & $47.3(31.9)$ & $81.5(135.8)$ & 0.49 \\
\hline $\begin{array}{c}\text { Remission }^{\circledR} \\
\text { Remission } \\
\text { Mild } \\
\text { Moderate } \\
\text { Severe }\end{array}$ & $\begin{array}{c}26(66.6) \\
11(28.2) \\
1(2.6) \\
1(2.6)\end{array}$ & $\begin{array}{c}33(84.6) \\
1(2.6)^{*} \\
3(7.7) \\
0\end{array}$ & $0.053^{\mathrm{b}}$ & & & & & & \\
\hline $\begin{array}{l}\text { HBI } \\
\text { remission } \\
\text { mild } \\
\text { moderate } \\
\text { severe }\end{array}$ & & & & $\begin{array}{c}18 \\
6 \\
1 \\
1\end{array}$ & $\begin{array}{c}21 \\
0^{*} \\
3 \\
0\end{array}$ & $0.050^{c}$ & & & \\
\hline $\begin{array}{c}\text { HBI, } \\
\text { median (IQR) } \\
\text { pMS } \\
\text { remission } \\
\text { mild } \\
\text { moderate } \\
\text { severe }\end{array}$ & & & & $3(2-5)$ & $2(1-4)$ & $0.104 \mathrm{~b}$ & $\begin{array}{l}8 \\
5 \\
0 \\
0\end{array}$ & $\begin{array}{c}12 \\
1 \\
0 \\
0\end{array}$ & $0.411^{\mathrm{b}}$ \\
\hline pMS median (IQR) & & & & & & & $1(0-2)$ & $0(0-1)$ & $0.117 \mathrm{c}$ \\
\hline SIBDQ & $50.8(8.3)$ & $54.7(8.9)$ & 0.001 & $51.9(7.5)$ & $57.3(7.8)$ & $<0.001$ & 48.8 & 50.1 & 0.55 \\
\hline Fatigue mean (SD) & $4.8(2.4)$ & $3.7(2.2)$ & 0.014 & $5.3(2.13)$ & $3.4(1.9)$ & 0.001 & $3.8(2.6)$ & $4.1(2.8)$ & 0.63 \\
\hline $\begin{array}{l}\text { Bodyweight mean } \\
\text { (SD), kg }\end{array}$ & $84.2(21.3)$ & $81.8(20.7)$ & 0.011 & $82.9(19.8)$ & $79.4(17.2)$ & $<0.001$ & $86.7(24.4)$ & $86.1(26.1)$ & 0.78 \\
\hline BMI mean (SD) & $27.7(5.8)$ & $26.9(5.4)$ & 0.006 & $27.5(5.9)$ & $26.4(5.2)$ & $<0.001$ & $28.2(5.9)$ & $27.9(5.9)$ & 0.61 \\
\hline HGS mean (SD), kg & $38.6(11.5)$ & $42.4(13.2)$ & $<0.001$ & $39.6(9.1)$ & $44.0(12.5)$ & $<0.001$ & $36.9(15.3)$ & $39.5(14.5)$ & 0.007 \\
\hline FRQoL & $73.6(19.6)$ & $75.9(21.2)$ & 0.47 & $71.4(17.1)$ & 74.5 (19.6) & 0.51 & $77.4(23.7)$ & $78.5(24.5)$ & 0.79 \\
\hline IBD-DI & $28.1(12.0)$ & $21.3(16.0)$ & 0.011 & $28.0(13.5)$ & $20.9(15.5)$ & 0.015 & $28.3(8.3)$ & $22.2(18.5)$ & 0.35 \\
\hline
\end{tabular}

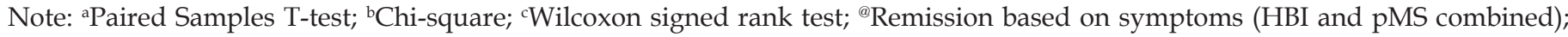
*2 missing values; BMI: Body Mass Index; HBI: Harvey-Bradshaw index; CRP: C-reactive Protein; FCP: Fecal Calprotectin; FRQOL: Food-Related Quality of Life; HGS: Handgrip Strength; IBD Inflammatory bowel disease; IBD-DI: IBD Disability Index; IQR: Interquartile Range; kg: Kilograms; MDSS: Mediterranean Diet Serving Score; No: Number; Pms: Partial Mayo score; SD standard Deviation; Sibdq: Short IBD questionnaire; y: years.

In the other patient the IBD medication had to be changed during the 6 months study period. This was in the patient with a FCP $>250 \mu \mathrm{g} / \mathrm{g}$ at baseline. We included him because he had a colonoscopy demonstrating endoscopic remission. However, 3.5 months after the start of the study he had a rectoscopy showing some ulcers. Biopsies showed a chronic, focally active inflammation with a granuloma. The patient was treated with suppositories of beclomethasone/5-ASA for 1 month. Mean QoL, as measured using the SIBDQ did also improve after the intervention (50.8 (SD 8.3) vs 54.7 (SD 8.9), $\mathrm{p}=0.001$ ). However, subgroup analyses revealed 
that this effect was only observed in the CD group (51.9 vs $57.3, \mathrm{p}$ $<0.001$; UC: 48.8 vs 50.1, $\mathrm{p}=0.55$ ). Participants lost body weight (84.2 vs $81.8 \mathrm{~kg}, \mathrm{p}=0.011$ ) resulting in a lower mean BMI after the intervention ( 27.7 vs $26.9, \mathrm{p}=0.006$ ). Importantly, muscle strength did not decrease in participants. The difference in MDSS between baseline and 6 months was correlated to the sIBDQ at 6 months $(r=$ $0.42, \mathrm{p}=0.023)$ and the HBI at 6 months $(\mathrm{r}=-0.613, \mathrm{p}=0.007)$, but not to the pMS, CRP or the severity of fatigue at 6 months.

\section{Discussion}

In this pilot study, a Mediterranean diet-based lifestyle intervention was well-tolerated in patients with quiescent IBD, as 27 out of 39 participants completed the intervention. Compared to dropouts, the completers reported a lower degree of disability and mean HBI, and a higher QoL at baseline. The Mediterranean diet-based lifestyle intervention improved mean dietary quality as reflected by an increase in MDSS. Both the SIBDQ and the IBD-DI improved after the intervention. We found that the adherence to the MeD-based lifestyle intervention was 69\%, with $65 \%$ actually improving their dietary quality. This is in line with other studies on MeD interventions. [20,21] studied the barriers and facilitators to introducing a MeD based lifestyle intervention, and found that nutritional support, knowledge and skills were facilitators, whereas obesogenic environment, life stressors, and demand for convenience were barriers [21].

When dropping-out participants in our study were asked why they dropped out, they frequently reported that the program took too much effort, or they cited a lack of time or motivation (for instance because of other dietary preferences of family members) Although dropouts experienced a higher level of disability, they did not report this as a reason to not complete the MeD-based lifestyle intervention. A recent large cohort study showed that adherence to a MeD protected against developing CD but not against UC [22]. In our study, we observed a mean improvement in QoL, disability, a trend for remission status and a decrease in CRP, but these improvements were due to the effects in patients with $\mathrm{CD}$. Chicco et al. [17] found that the improvements in IBD specific outcomes were similar between CD and UC patients [23]. The fact that in our study the improvements were only observed in CD patients is probably due to the fact that the improvement in dietary quality was most outspoken in CD patients. Our intervention led to a reduction in body weight and BMI. This is most likely due to loss of fat mass, as the handgrip strength increased between baseline and end of follow up. Chicco et al. [17] found a similar effect on BMI and a reduction in waist circumference in response to their MeD intervention. This suggests that the body weight lost is predominantly due to loss of visceral fat. This appears to be of special benefit in CD patients, as the ratio between visceral and subcutaneous fat is an indicator of a complicated disease phenotype [24].

The beneficial effect of enteral nutrition on remission status in CD is well known, but the practical applicability is low, due to its unpalatability or the presence of the nasogastric tube. [1] A number of oral dietary treatments has been introduced in recent years $[25,26]$ of which the Crohn's disease exclusion diet (CDED) best resembles a 'normal' oral diet. The premise on which the CDED was based is that CD is caused by mucosal and translocating bacteria. It was developed by excluding foods that have been associated with a diminished host barrier and less bacterial clearance, resulting in dysbiosis allowing bacteria to translocate [27]. Similar to our Mediterranean diet-based lifestyle intervention, the CDED includes the intake of fiber, vegetables, fruits, legumes, olive oil, poultry and fish, whereas alcohol, soft drinks and processed foods such as candy, cookies and chips are not allowed [28]. However, there are some differences between our MeD and CDED. In CDED dairy products were not allowed, whereas in our MeD-based lifestyle intervention full-fat yoghurt, small amount of cheeses and cottage cheese were allowed. We discouraged the intake of refined carbohydrates whereas in the CDED white rice and rice noodles were allowed. Whether these differences are important or that the reduction in consuming a Western diet rich in processed and industrialised foods is the main factor leading to the beneficial effects observed in both our Mediterranean diet-based lifestyle intervention and CDED, is unknown. In this pilot study we did not investigate the immunological responses to the MeD, nor did we look into the possible changes in the microbiome. The sample size of our pilot study was small, our group was heterogeneous and we did not include a control group. Therefore, caution should be taken when interpreting the presented results. Our primary goal was to investigate whether patients with IBD would be able to tolerate and adhere to the Mediterranean diet-based lifestyle intervention.With the number of patients we included, we were able to demonstrate an adherence rate of $69 \%$. Without a control group we cannot confirm that our observed improvements were only established due to the Mediterranean diet-based lifestyle intervention. However, the improvement in dietary quality was correlated to the SIBDQ and HBI at 6 months. Furthermore, only 1 patient had a flare of his disease 3.5 months after the start of the study, requiring additional medical therapy. But this was also the patient with a FCP $>250$ at baseline. We included him because he had a colonoscopy demonstrating endoscopic remission. In hindsight, we should not have included this patient. The heterogeneity of our group could make it difficult to interpreted the results. However, we can conclude that IBD patients in remission tolerated the intervention and most of them were able to adhere to the entire program. In addition, this study 
is the first performed in Northern European IBD patients where a Mediterranean diet is not the standard diet.

\section{Conclusion}

Our study underlines the importance of nutrition in the management of IBD. In quiescent IBD patients, $69 \%$ was able to adhere to the Mediterranean diet-based lifestyle intervention. The improvement in MDSS was correlated to the increase in QoL, but was only observed in patients with CD probably because the improvement in dietary quality was most outspoken in these patients. CRP and BMI decreased during the study period. Larger trials with a control group are needed to investigate whether the reported effects are sustained over a longer period of time, and the effects of the Mediterranean diet-based lifestyle intervention on microbiome diversity, intestinal barrier function and systemic inflammation.

\section{References}

1. Ilse Molendijk, Sander van der Marel, PW Jeroen Maljaars (2019) Towards a Food Pharmacy: Immunologic Modulation through Diet. Nutrients 11(6): 1239.

2. Kinsey L, S Burden, et al. (2016) A survey of people with inflammatory bowel disease to investigate their views of food and nutritional issues. Eur J Clin Nutr 70(7): 852-854.

3. Casanova MJ, Maria Chaparro, Begona Molina, Olga Merino, Ricardo Batanero, et al. (2017) Prevalence of Malnutrition and Nutritional Characteristics of Patients with Inflammatory Bowel Disease. J Crohns Colitis 11(12): 1430-1439.

4. Ananthakrishnan AN, Hamed Khalili, Mingyang Song, Leslie M Higuchi, James M Richter, et al. (2015) High School Diet and Risk of Crohn's Disease and Ulcerative Colitis. Inflamm Bowel Dis 21(10): 2311-2319.

5. Guerreiro CS, Paula Ferreira, Lourdes Tavares, Paula Moura Santos, Manuela Neves, et al. (2009) Fatty acids, IL6, and TNFalpha polymorphisms: an example of nutrigenetics in Crohn's disease. Am J Gastroenterol 104(9): 2241-2249.

6. Tanaka M, Yasushi Iwao, Satoshi Sasaki, Susumu Okamoto, Haruhiko Ogata, et al. (2007) Moderate dietary temperance effectively prevents relapse of Crohn disease: a prospective study of patients in remission. Gastroenterol Nurs 30(3): 202-210.

7. Calder PC (2006) n-3 polyunsaturated fatty acids, inflammation, and inflammatory diseases. Am J Clin Nutr 83(6): 1505-1519.

8. Ana Gutiérrez, Pedro Zapater, Oriol Juanola, Laura Sempere, Marifé García, et al. (2016) Gut Bacterial DNA Translocation is an Independent Risk Factor of Flare at Short Term in Patients with Crohn's Disease. Am J Gastroenterol 111(4): 529-540.

9. Chang J, Rupert W Leong, Valerie C Wasinger, Matthew Ip, Michael Yang, et al. (2017) Impaired Intestinal Permeability Contributes to Ongoing Bowel Symptoms in Patients With Inflammatory Bowel Disease and Mucosal Healing. Gastroenterology 153(3): 723-731.

10. Wolfgang Scheppach, Frank Weiler (2004) The butyrate story: old wine in new bottles? Curr Opin Clin Nutr Metab Care 7(5): 563-567.

11. Kaser A (2010) Inflammatory bowel disease. Annu Rev Immunol 28: 573-621.

12. Kleinewietfeld M, Arndt Manzel, Jens Titze, Heda Kvakan, Nir Yosef, et al. (2013) Sodium chloride drives autoimmune disease by the induction of pathogenic TH17 cells. Nature 496(7446): 518-22.
13. Lee D, Lindsey Albenberg, Charlene Compher, Robert Baldassano , David Piccoli, et al. (2015) Diet in the pathogenesis and treatment of inflammatory bowel diseases. Gastroenterology 148(6): 1087-106.

14. Galilea-Zabalza I, Pilar Buil-Cosiales, Jordi Salas-Salvadó, Estefanía Toledo, Carolina Ortega-Azorín, et al. (2018) Mediterranean diet and quality of life: Baseline cross-sectional analysis of the PREDIMED-PLUS trial. Plos one 13(6): 0198974

15. Evdokia K Mitsou, Aimilia Kakali, Smaragdi Antonopoulou, Konstantinos C Mountzouris, Mary Yannakoulia, et al. (2017) Adherence to the Mediterranean diet is associated with the gut microbiota pattern and gastrointestinal characteristics in an adult population. Br J Nutr 117(12): 1645-1655.

16. Forsyth C, Matina Kouvari, Nathan M D'Cunha, Ekavi N Georgousopoulou, Demosthenes B Panagiotakos, et al. (2018) The effects of the Mediterranean diet on rheumatoid arthritis prevention and treatment: a systematic review of human prospective studies. Rheumatol Int 38(5): 737-747.

17. Chicco F, Salvatore Magrì, Arianna Cingolani, Danilo Paduano, Mario Pesenti, et al. (2021) Multidimensional Impact of Mediterranean Diet on IBD Patients. Inflamm Bowel Dis 27(1): 1-9.

18. Efstathia Papada, Charalampia Amerikanou, Alastair Forbes, Andriana C Kaliora (2020) Adherence to Mediterranean diet in Crohn's disease. Eur J Nutr 59(3): 1115-1121.

19. Josip Vrdoljak, Marino Vilović, Piero Marin Živković, Ivana Tadin Hadjina, Doris Rušić et al. (2020) Mediterranean Diet Adherence and Dietary Attitudes in Patients with Inflammatory Bowel Disease. Nutrients 12(11): 3429.

20. Rosario Alonso-Domínguez, Luis García-Ortiz, Maria C Patino-Alonso, Natalia Sánchez-Aguadero, Manuel A Gómez-Marcos, et al. (2019) Effectiveness of A Multifactorial Intervention in Increasing Adherence to the Mediterranean Diet among Patients with Diabetes Mellitus Type 2: A Controlled and Randomized Study (EMID Study) Nutrients 11(1): 162.

21. Haigh L, Susan Bremner, David Houghton, Elsbeth Henderson, Leah Avery, et al. (2019) Barriers and Facilitators to Mediterranean Diet Adoption by Patients With Nonalcoholic Fatty Liver Disease in Northern Europe. Clin Gastroenterol Hepatol 17(7): 1364-1371.

22. Khalili H, Niclas Hakansson, Simon S Chan, Ye Chen, Paul Lochhead, et al. (2020) Adherence to a Mediterranean diet is associated with a lower risk of later-onset Crohn's disease: results from two large prospective cohort studies. Gut 69(9): 1637-1644.

23. Chicco F, Salvatore Magrì, Arianna Cingolani, Danilo Paduano, Mario Pesenti, et al. (2020) Multidimensional Impact of Mediterranean Diet on IBD Patients. Inflammatory bowel diseases 27(1): 1-9.

24. Erhayiem B, Rajpal Dhingsa, Christopher J Hawkey, Venkataraman Subramanian, et al. (2011) Ratio of visceral to subcutaneous fat area is a biomarker of complicated Crohn's disease. Clin Gastroenterol Hepatol 9(8): 684-887.

25. Svolos V, Richard Hansen, Ben Nichols, Christopher Quince, Umer Z Ijaz, et al. (2019) Treatment of Active Crohn's Disease With an Ordinary Foodbased Diet That Replicates Exclusive Enteral Nutrition. Gastroenterology 156(5): 1354-1367.

26. Boneh RS, Johan Van Limbergen, Eytan Wine, Amit Assa, Ron Shaoul, et al. (2020) Dietary Therapies Induce Rapid Response and Remission in Pediatric Patients With Active Crohn's Disease. Clin Gastroenterol Hepatol 19(4): 752-759.

27. Arie Levine, Rotem Sigall Boneh, Eytan Wine (2018) Evolving Role of Diet in the Pathogenesis and Treatment of Inflammatory Bowel Diseases. Gut 67(9)-1726-1738.

28. Rotem Sigall Boneh, Tamar Pfeffer Gik, Idit Segal, Tsili Zangen, Mona Boaz, et al. (2014) Partial enteral nutrition with a Crohn's disease exclusion diet is effective for induction of remission in children and young adults with Crohn's disease. Inflamm Bowel Dis 20(8): 1353-1360. 